\title{
Diagnostic yield of EUS-guided through-the-needle microforceps biopsy versus EUS-FNA of pancreatic cystic lesions: a systematic review and meta-analysis
}

\section{(ㄷ)(1) $(-)$}

\author{
Authors \\ Institutions \\ 1 Department of Internal Medicine, University of Florida, \\ Gainesville, Florida, United States \\ 2 Division of Gastroenterology and Hepatology, University \\ of Florida, Gainesville, Florida, United States
}

Donevan R. Westerveld ${ }^{1}$, Sandeep A. Ponniah ${ }^{1}$, Peter V. Draganov², Dennis Yang ${ }^{2}$ in PCs. submitted 22.10.2019

accepted after revision 15.1 .2020

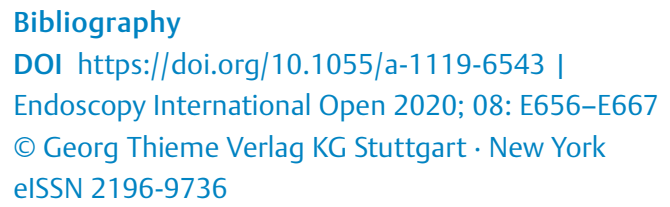

Corresponding author

Dennis Yang, 1329 SW 16th, Street, Room \#5252, Gainesville, FL 32608

Fax: +1-352-627-9002

dennis.yang@medicine.ufl.edu

丹 Supplementary material

Online content viewable at:

https://doi.org/10.1055/a-1119-6543

\section{ABSTRACT}

Background and study aims Accurate diagnosis and risk stratification of pancreatic cysts (PCs) is challenging. The aim of this study was to perform a systematic review and meta-analysis to assess the feasibility, safety, and diagnostic yield of endoscopic ultrasound-guided through-theneedle biopsy (TTNB) versus fine-needle aspiration (FNA)

Methods Comprehensive search of databases (PubMed, EMBASE, Cochrane, Web of Science) for relevant studies on TTNB of PCs (from inception to June 2019). The primary outcome was to compare the pooled diagnostic yield and concordance rate with surgical pathology of TTNB histology and FNA cytology of PCs. The secondary outcome was to estimate the safety profile of TTNB.

Results: Eight studies (426 patients) were included. The diagnostic yield was significantly higher with TTNB over FNA for a specific cyst type (OR: 9.4; $95 \% \mathrm{Cl}$ : [5.7-15.4]; $\mathrm{I}^{2}$ $=48$ ) or a mucinous cyst (MC) (OR: 3.9 ; $95 \%$ Cl: [2.0-7.4], I ${ }^{2}$ $=72 \%)$. The concordance rate with surgical pathology was significantly higher with TTNB over FNA for a specific cyst type (OR: 13.5 ; $95 \% \mathrm{Cl}$ : [3.5-52.3]; $\mathrm{I}^{2}=48$ ), for a MC (OR: 8.9; 95\% [Cl: 1.9-40.8]; $\left.\mathrm{I}^{2}=29\right)$, and for $\mathrm{MC}$ histologic severity (OR: $10.4 ; 95 \% \mathrm{Cl}$ : [2.9-36.9]; $\mathrm{I}^{2}=0$ ). The pooled sensitivity and specificity of TTNB for MCs were $90.1 \%$ (95\% Cl: [78.4-97.6]; $\left.\mathrm{I}^{2}=36.5 \%\right)$ and $94 \%(95 \% \mathrm{Cl}$ : [81.599.7]; $\left.\left.\right|^{2}=0\right)$, respectively. The pooled adverse event rate was $7.0 \%$ (95\% Cl: [2.3-14.1]; $\left.\mathrm{I}^{2}=82.9\right)$.

Conclusions TTNB is safe, has a high sensitivity and specificity for MCs and may be superior to FNA cytology in riskstratifying MCs and providing a specific cyst diagnosis.

\section{Introduction}

Pancreas cysts (PCs) are being diagnosed with increasing frequency given the widespread use of cross-sectional imaging in our health care system [1]. PCs can be broadly categorized as either non-neoplastic or neoplastic, with the latter being estimated as high as $13.5 \%$ in the general population [2]. Accurate diagnosis and risk stratification of neoplastic cysts are crucial as to provide guidance for the most appropriate management strategy.
The optimal diagnostic approach to PCs remains unclear as there is currently no single test that can reliably differentiate non-neoplastic PCs from those lesions with malignant potential or harboring malignancy. Given the well-recognized limitations of imaging alone [2-4], EUS-guided fine-needle aspiration (EUS-FNA) for cytology and cyst fluid analysis is the commonly performed guideline endorsed test for aid with diagnosis and risk stratification [4-10]. FNA cytology is often limited by the scant cellularity within the cyst fluid $[11,12]$. While cyst fluid carcinoembryonic antigen (CEA) has been traditionally used to differentiate mucinous versus non-mucinous PCs [13], it has 
modest diagnostic accuracy and does not discriminate between benign and malignant mucinous cysts $[14,15]$.

Recently, a through-the-needle microforceps device (Moray Microforceps, US Endoscopy, Mentor, Ohio, United States) was introduced for EUS-guided tissue sampling of PCs. The microforceps can be advanced through the lumen of a standard 19gauge EUS-FNA needle for through-the-needle tissue biopsy (TTNB) of PCs. Since its introduction, there have been several studies reporting on the performance of the TTNB [16-28]. We performed a systematic review and meta-analysis of the current literature to better assess the feasibility, safety, and diagnostic yield of TTNB as compared to EUS-FNA in the evaluation of PCs.

\section{Methods}

\section{Search strategy}

We conducted a comprehensive search of four electronic databases (MEDLINE through Pubmed, EMBASE, Cochrane, and Web of Science) for all relevant studies with the last search performed in July 2019. The search was performed with the assistance of an experienced medical librarian.

The PRISMA (Preferred Reporting Items for Systematic Reviews and Meta-Analyses) guidelines were followed. Key terms used in the search query included a combination of the following: "Endoscopic ultrasound-guided through the needle microforceps", "microforceps", "micro-forcep”, "pancreas AND microforceps", "through the needle", ("pancreas AND through the needle,") ("pancreas" AND "through the needle" AND "EUS"), ("pancreatic cysts" AND "through the needle"), ("pancreatic cyst" AND "moray"), "moray micro-forceps," ("microforceps" AND "pancreas”). We attempted to identify additional studies by reviewing the reference list of all included studies and by manual search in order to retrieve other articles that may have been missed on the initial search strategy. Using the predefined inclusion and exclusion criteria, two investigators (S.P., D.W.) independently screened the title and abstract of all studies identified in the primary search. The full text of all relevant studies was subsequently reviewed. Discrepancies were resolved by consensus and by discussion with the co-authors (P.V.D., D.Y.).

\section{Study selection}

All studies were screened based on the predefined inclusion and exclusion criteria. Studies were included if they: (1) were retrospective or prospective, case-control or cohort studies and clinical trials (including randomized controlled trials); (2) reported EUS-TTNB of pancreas cysts; and (3) included data on cytology and/or histology for EUS-TTNB. Studies were excluded if: (1) they included fewer than five cases; (2) animal studies; (3) EUS-TTNB was performed in non-cystic pancreatic lesions, (3) cytologic or histological diagnosis for EUS-TTNB was not provided; or (4) were publications in a language other than English.

In the case of publications from the same group of authors, we contacted the authors as to determine whether these were from the same cohort. If publications were from the same cohort or if this could not be verified, then only data from the most recent and/or most comprehensive study was included. In our search query, we encountered one such study by Kovacevic et al [20].

\section{Data extraction and quality assessment}

Three investigators (S.P., D.W., D.Y.) independently extracted data from each study by using a standardized data collection form, which included: (1) study author; (2) year of publication; (3) study period; (4) patient demographics; (5) pancreatic cyst characteristics (i.e. size, morphology, location); (6) cyst fluid analysis; (7) cytology from EUS-FNA; (8) histology from EUSTTNB (9) adverse events (AES); and (10) surgical pathology.

Contingency $2 \times 2$ tables were constructed with information of true positive, false positive, true negative, and false negative for TTNB discrimination between mucinous and non-mucinous cysts, with surgical pathology as the reference standard.

The quality of the studies was assessed by using a modified Newcastle Ottawa Scale (NOS) for cohort studies [29]. The modified NOS consisted of 6 components: representative of the average population ( 1 point for population-based studies, 0.5 point for multi-center studies, 0 point for single-center hospital-based study), large cohort size ( 1 point $>50$ patients, 0.5 point for 30-49 patients, and 0 point $<30$ patients); information on how a mucinous cyst was diagnosed ( 1 point if reported with clarity; 0 point if not reported); information on the specific cyst diagnosis obtained by FNA and TTNB (1 point if reported for both; 0.5 if reported for either FNA or TTNB, 0 point if not reported); information on AEs ( 1 point if reported, 0 point if not reported); information on concordance between FNA, TTNB and surgical pathology ( 1 point if reported, 0.5 if reported for either FNA or TTNB, 0 point if not reported). Studies with scores $\geq 5,3-4$, and $\leq 2$ were suggestive of high, moderate, and low quality, respectively.

\section{Study aim and definitions}

The primary aim of this study was to perform a systematic review and meta-analysis to assess the feasibility, safety, and diagnostic yield of EUS-TTNB versus FNA in PCs. A secondary aim was to determine the pooled sensitivity and specificity of TTNB for mucinous cysts. Technical feasibility was defined as successful passage of the microforceps through the indwelling EUS needle and completion of intended tissue acquisition (EUSTTNB). Diagnostic yield was defined as the number of cases in which a diagnosis was attained divided by the total of number of cases. The concordance rate was defined as the number of cases in which TTNB or FNA matched the final surgical diagnosis divided by the total number of surgical specimens for that category. TTNB diagnostic accuracy parameters for mucinous cysts were calculated from eligible studies by using the surgical pathology as the reference standard. AEs were defined as any deviation from the expected post-procedural clinical course. Intracystic hemorrhage was defined as the presence of blood within a cyst as identified on EUS or subsequent cross-sectional imaging. 


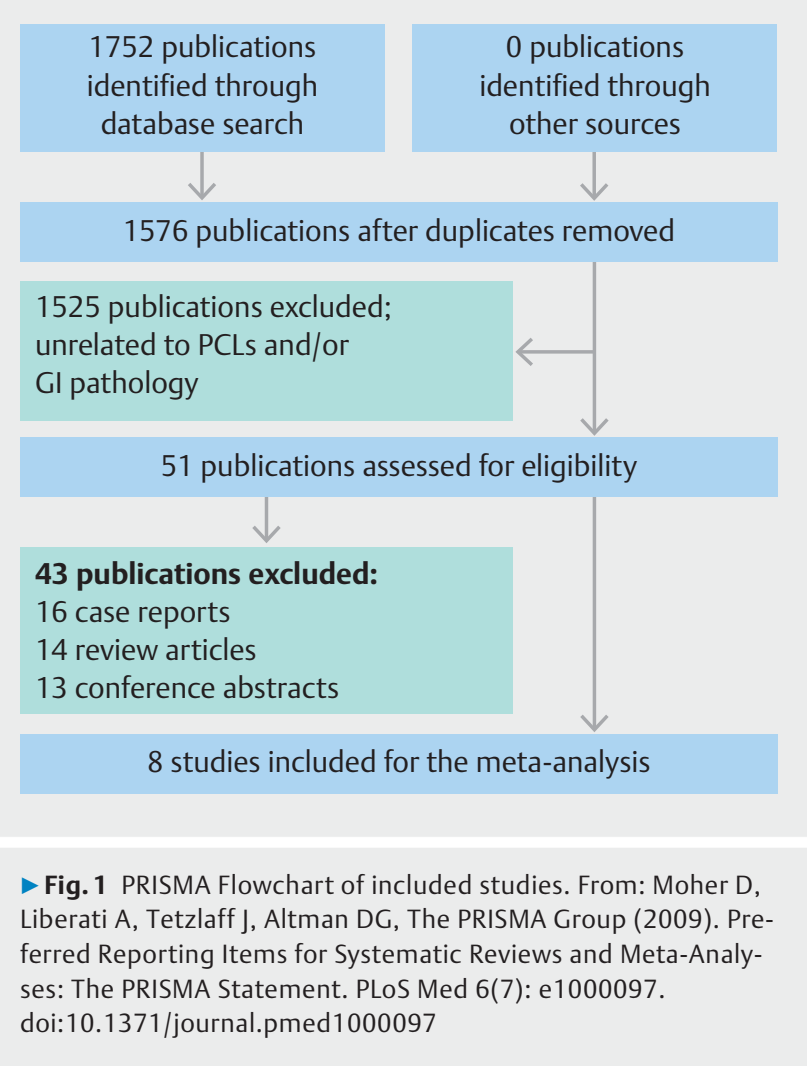

\section{Statistical analysis}

The data was analyzed with Review Manager 5.2 (RevMan, The Nordic Cochrane Center, The Cochrane Collaboration, 2012) for computation of comparative forest plots and funnel plots. Pooled proportions were calculated from the weighted means of individual proportions and funnel plots were calculated using STATA (Stata Statistical Software, STATA 15, College Station, Texas, United States). Subgroup analysis were also performed to compare EUS-TTNB verses EUS-FNA and reported as odds ratios (OR) with $95 \%$ confidence intervals $(\mathrm{Cl})$ calculated using the Mantel-Haenszel method. The heterogeneity estimate of the studies was calculated using the Cochran $\mathrm{Q}$ test $\mathrm{I}^{2}$ statistic. Values of $<30 \%, 30 \%$ to $60 \%, 61 \%$ to $75 \%$, and $>75 \%$ were considered low, moderate, substantial, and considerable heterogeneity, respectively [30]. A fixed- or random-effects model was applied based on heterogeneity. In the eligible studies, the pooled sensitivity and specificity was calculated. A receiver operating characteristics (ROC) curve could not be calculated as none of the studies included reported false positive results for TTNB in the diagnosis of mucinous cysts. Funnel plots were visually inspected for publication bias; Egger's test was not performed to test for funnel plot asymmetry as this is not appropriate for meta-analysis with less than 10 studies [31-33].

\section{Results}

\section{Search results}

Our primary search strategy yielded 1752 studies, of which 176 were duplicates. Of the remaining 1576 publications, 1525
Study

Percent

ID $(95 \% \mathrm{Cl})$

Barresi et al, 2018

Basar et al, 2018

Crino et al, 2019

Kovacevic et al, 2019

Mittal et al, 2018

Yang et al, 2018

Yang et al, 2019

Zhang et al, 2018

FE Overall $\left(I^{2}=45.5 \%\right)$

Overall 0.982 (95\% Cl: $0.968,0.993)\left(I^{2}=45.5 \%\right)$

$\begin{array}{llllll}0 & .2 & .4 & .6 & .8 & 1\end{array}$

- Fig. 2 Pooled technical success of EUS-TTNB in the evaluation of pancreatic cysts (Fixed-effect Model).

were excluded after screening titles and abstracts. Full text review was subsequently performed on 51 studies using our predefined inclusion and exclusion criteria. Eight studies with a total of 426 cases were included in the final meta-analysis [2128]. The study flow diagram is shown in $>$ Fig. 1 .

\section{Study characteristics and quality assessment}

Study characteristics are summarized in $>$ Table 1 . All articles were cohort studies ( 7 retrospective and 1 prospective), study period between 2014 to 2018, and originating from the United States $(n=5)$ or Europe $(n=3)$. The mean age ranged between 50 to 70 years with $59.9 \%$ ( 255 out of 426 ) being female. Most studies reported a mean pancreas cyst size between $28.2 \mathrm{~mm}$ to $40.7 \mathrm{~mm}$. Pancreas cyst location was reported in all studies except by Kovacevic et al [19]. Most cysts were primarily located in the body/tail (64.6\%) followed by head/uncinated process (35.4\%). The median number of passes for EUS-TTNB was three, with two studies reporting tissue acquisition until a visible specimen was obtained $[22,26]$.

The quality of the studies was assessed by using the modified NOS scale as shown in $>$ Table 2 . None of the studies were population based; with equal number of single center $(n=4)$ and multicenter $(n=4)$ studies. Three studies had $>50$ patients $[21,27,28]$, four studies included $30-49$ patients $[22,23,25$, 26 , and one study $<30$ patients [24]. Six out of the eight studies provided clear definitions on how a mucinous cyst was diagnosed $[21,22,25-28]$. Six of the 8 studies provided information on the specific cyst type diagnosis obtained via TTNB and FNA [22-27]. All studies provided information on adverse events. Six of the eight studies provided information on FNA cytology, TTNB histology and the corresponding surgical pathology. Overall, three studies were considered to be of high quality 


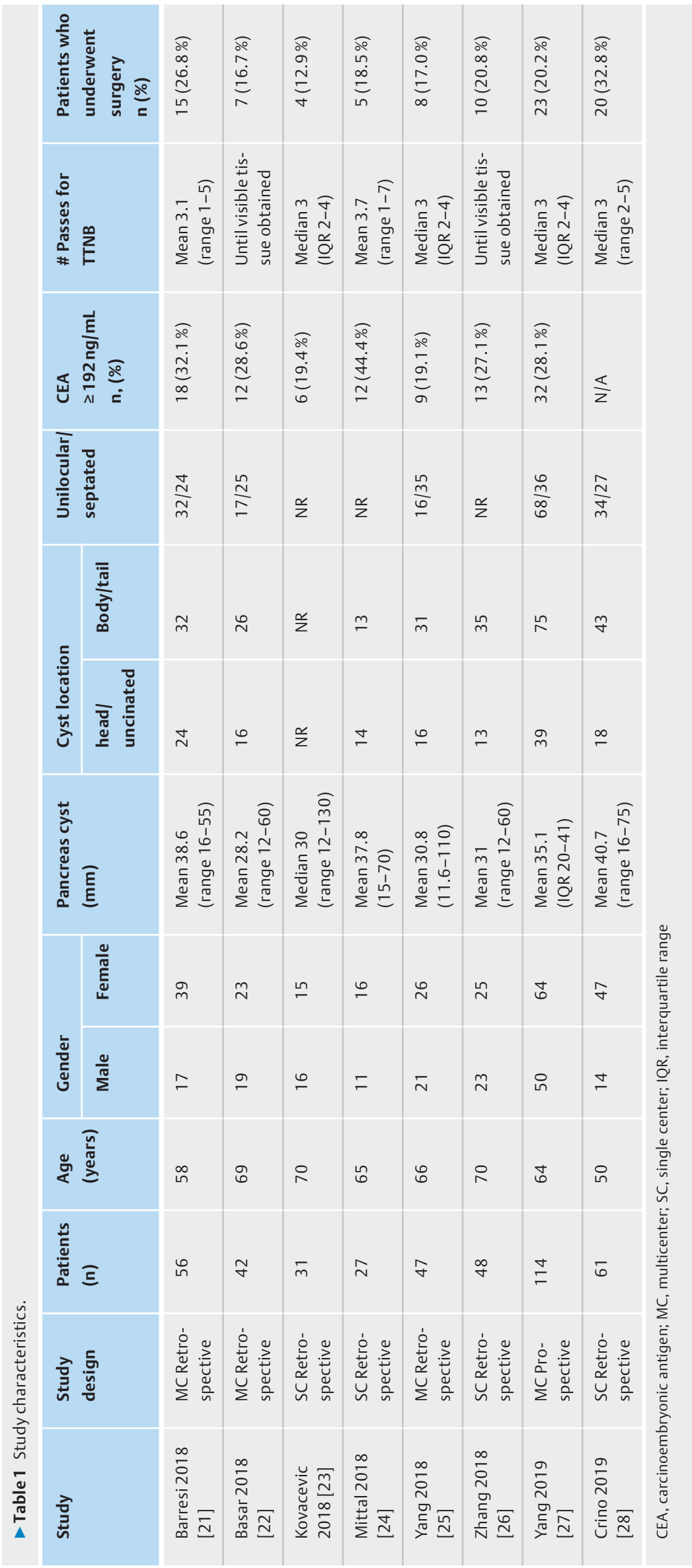


- Table 2 Study quality assessment using the modified Newcastle Ottawa Scale.

\begin{tabular}{|c|c|c|c|c|c|c|c|}
\hline Study & $\begin{array}{l}\text { Representa- } \\
\text { tive of aver- } \\
\text { age popula- } \\
\text { tion }\end{array}$ & Cohort Size & $\begin{array}{l}\text { Information } \\
\text { on how a } \\
\text { mucinous } \\
\text { cyst was } \\
\text { diagnosed }\end{array}$ & $\begin{array}{l}\text { Information } \\
\text { on specific } \\
\text { diagnosis } \\
\text { by FNA and } \\
\text { TTNB }\end{array}$ & $\begin{array}{l}\text { Information } \\
\text { on adverse } \\
\text { events }\end{array}$ & $\begin{array}{l}\text { Data on } \\
\text { TTNB, FNA } \\
\text { and cor- } \\
\text { responding } \\
\text { surgical } \\
\text { pathology }\end{array}$ & Total \\
\hline Barresi 2018 [21] & 0.5 & 1 & 1 & 0.5 & 1 & 0.5 & 4.5 \\
\hline Basar 2018 [22] & 0.5 & 0.5 & 1 & 1 & 1 & 1 & 5 \\
\hline Kovacevic 2018 [23] & 0 & 0.5 & 0.5 & 1 & 1 & 1 & 4 \\
\hline Mittal 2018 [24] & 0 & 0 & 0.5 & 1 & 1 & 1 & 3.5 \\
\hline Yang 2018 [25] & 0.5 & 0.5 & 1 & 1 & 1 & 1 & 5 \\
\hline Zhang 2018 [26] & 0 & 0.5 & 1 & 1 & 1 & 0 & 3.5 \\
\hline Yang 2019 [27] & 0.5 & 1 & 1 & 1 & 1 & 1 & 5.5 \\
\hline Crino 2019 [28] & 0 & 1 & 1 & 0.5 & 1 & 1 & 4.5 \\
\hline
\end{tabular}

$[22,25,28]$, five of medium quality $[21,23,24,26,28]$, and none of low quality.

\section{Feasibility of EUS-TTNB in the evaluation of pancreatic cysts}

In all, EUS-TTNB was successfully performed in 418 of 426 cases, for a pooled technical success of $98.2 \%$ (95\% Cl: [96.8-

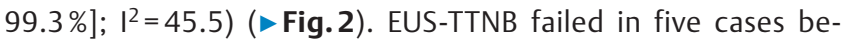
cause the microforceps could not be advanced through the angulated EUS-FNA needle via a transduodenal approach $[23,25]$. In the remainder three cases, EUS-TTNB was not performed due to the presence of interposing vessels between the EUS needle and cyst $(n=1)$, as a result of insufficient sedation and transient hypoxia prior to EUS-TTNB $(n=1)$, or in the setting of a bloody aspirate with EUS-FNA $(n=1)[25,28]$.

\section{Diagnostic yield of TTNB versus FNA in pancreas cysts}

\section{Specific cyst type}

The specific cyst type diagnosis obtained via TTNB histology and FNA cytology from all the studies in this meta-analysis are summarized in $>$ Table 3 . The pooled diagnostic yield for a specific cyst type was significantly higher with TTNB histology (72.5\%; 95\% Cl: [60.6-83.0]) than FNA cytology (38.1\%; 95\% $\mathrm{Cl}$ : [18.0-60.5]). Furthermore, in comparator analysis the diagnostic yield was significantly higher with TTNB compared to FNA (OR: 9.37; $95 \%$ Cl: [5.69-15.42]), with moderate heterogeneity among the studies $\left(I^{2}=48\right)(\vee$ Fig. 3 ).

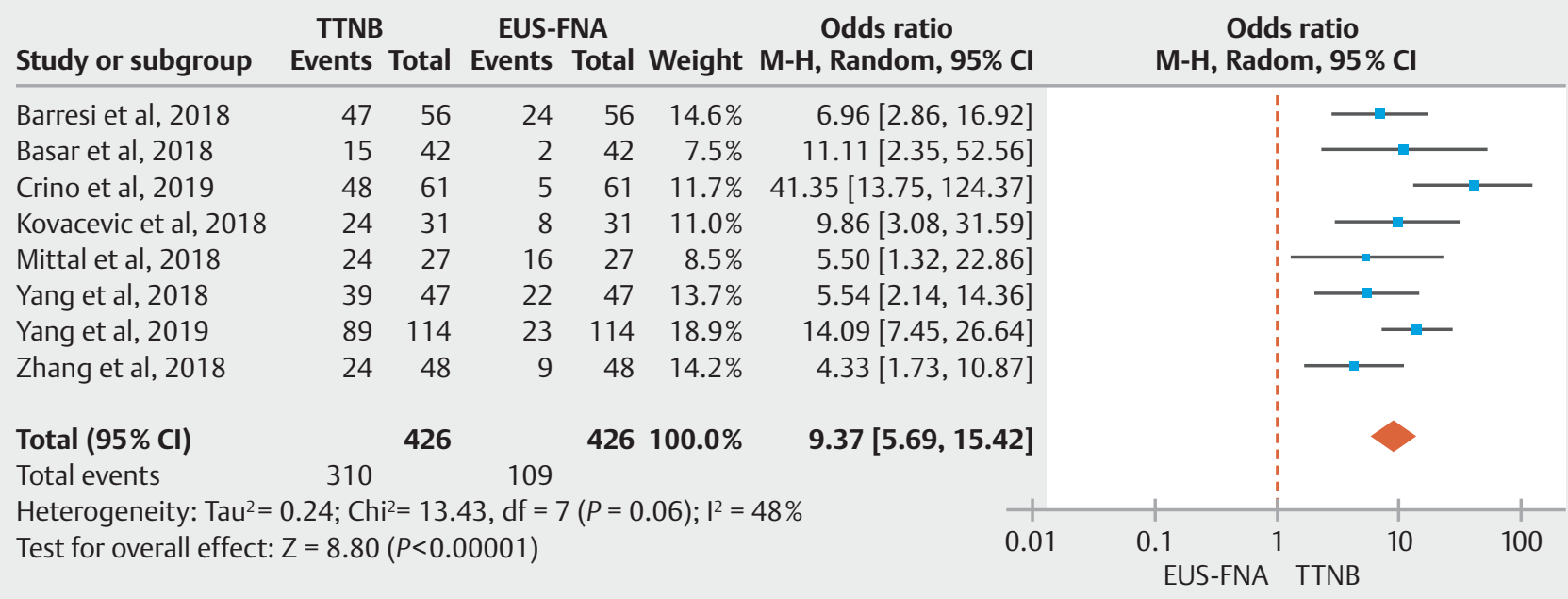

- Fig. 3 Pooled diagnostic yield of TTNB histology vs FNA cytology for a specific cyst type diagnosis (Random-Effect Model). 


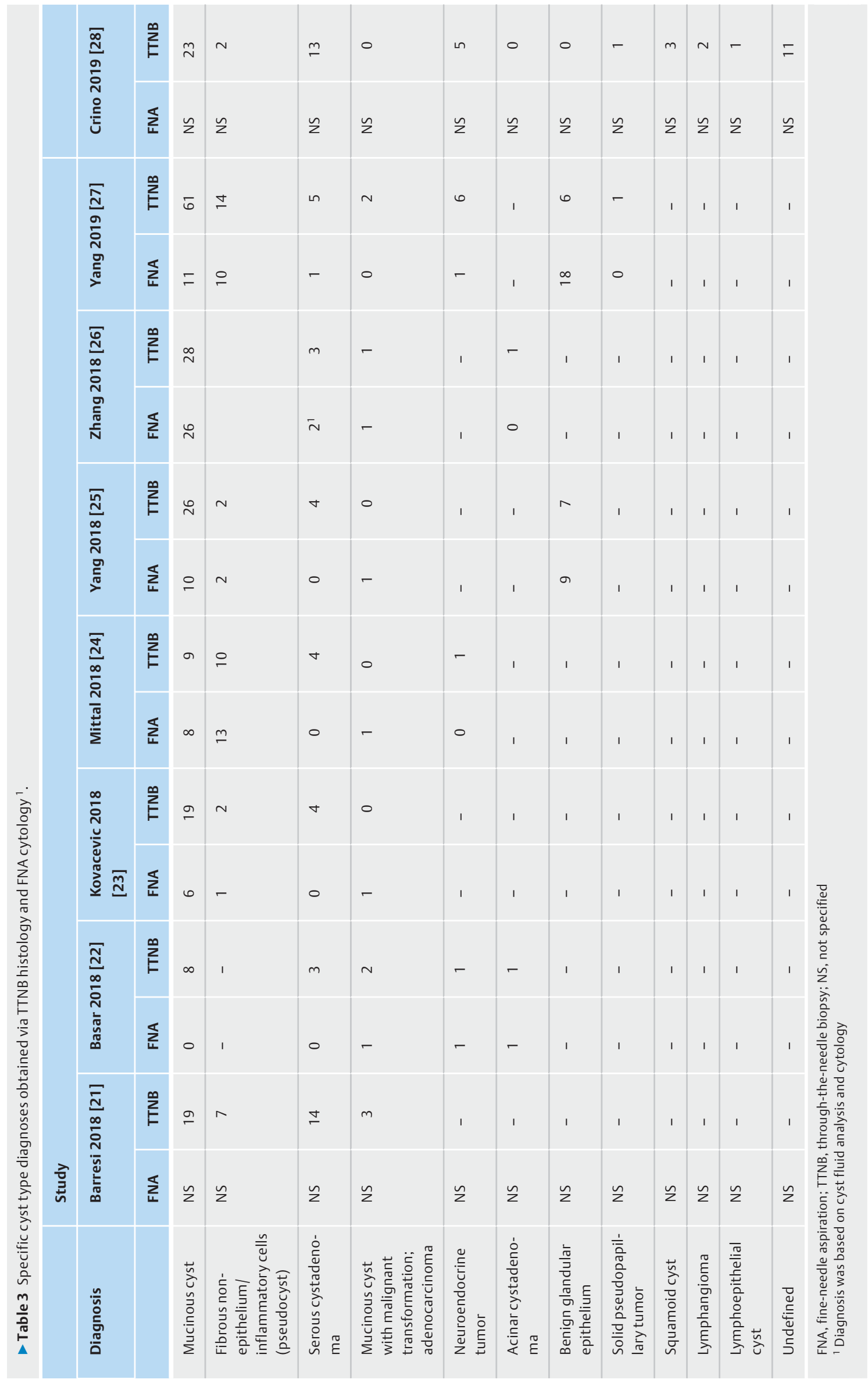




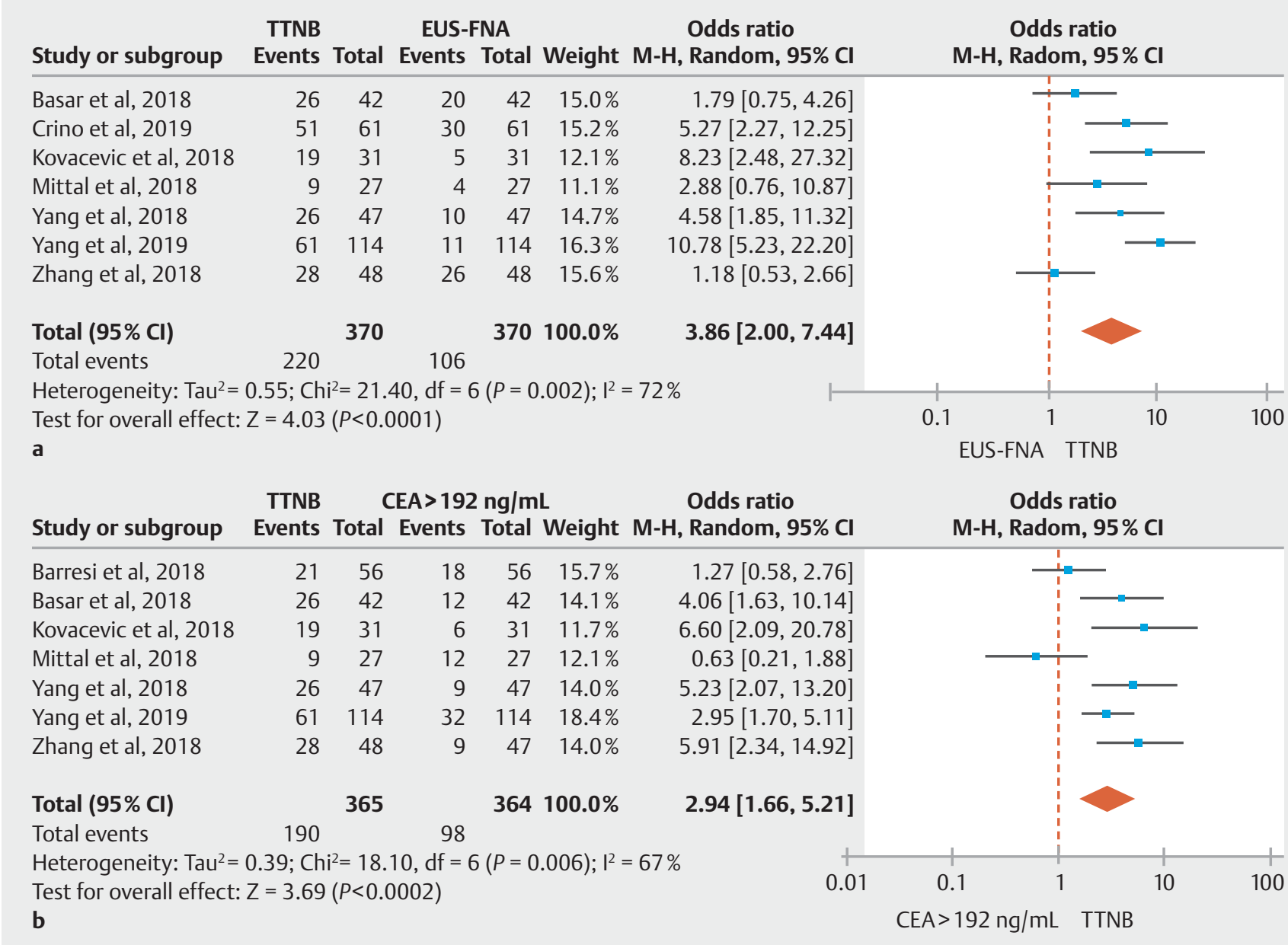

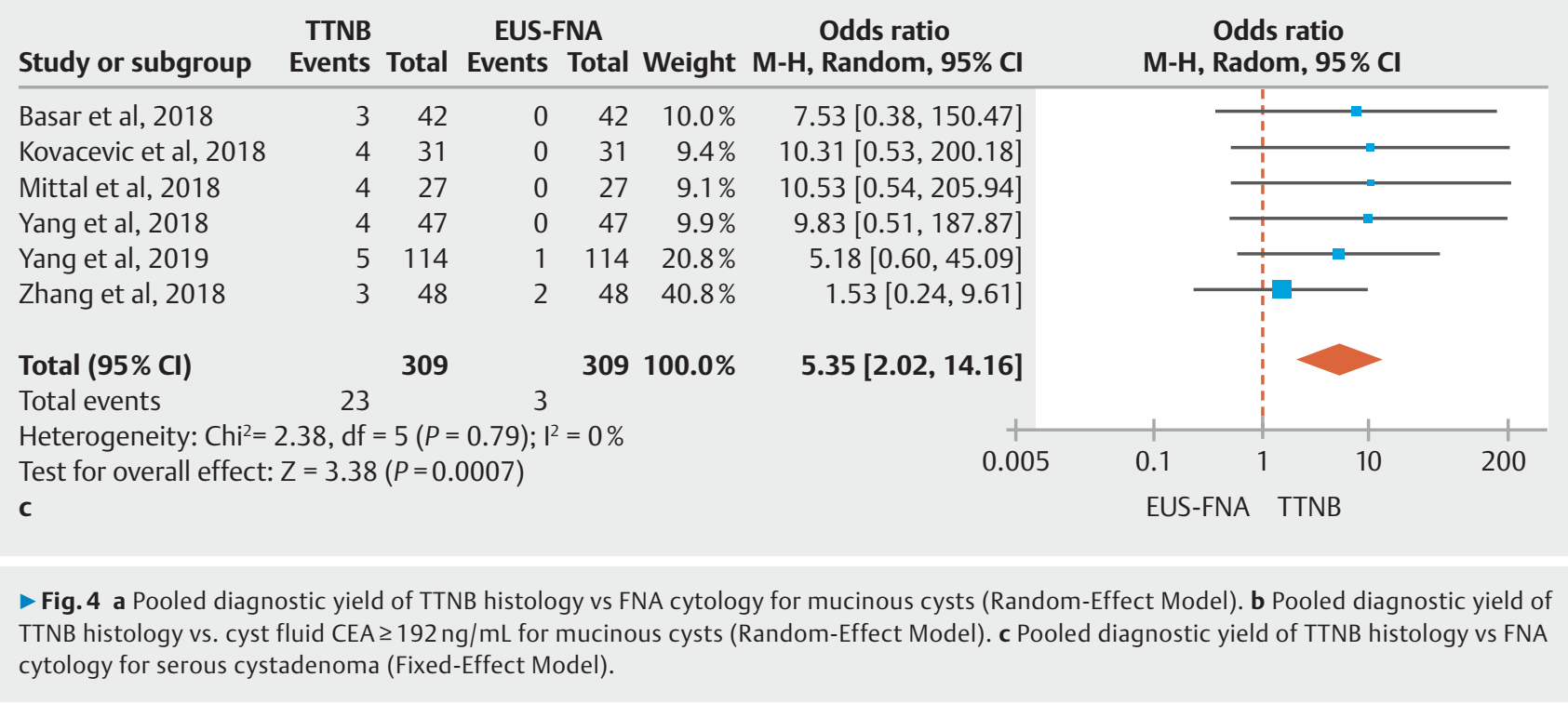

\section{Mucinous cysts}

The pooled diagnostic yield for a mucinous cyst was $56.2 \%$ (95\% Cl: [45.1-67.0]) with TTNB and 29.5\% (95\% Cl: [15.545.9]) with FNA, respectively. Cyst fluid CEA levels were specified in 7 out of the 8 studies [21-27]. The pooled rate of PCs with CEA $\geq 192 \mathrm{ng} / \mathrm{mL}$ was $28.2 \%$ (95\% Cl: [23.7-32.8]). Over- all, in comparator analysis the diagnostic yield for a mucinous cyst was significantly higher with TTNB histology when compared to either FNA cytology (OR: 3.86; $95 \% \mathrm{Cl}$ : [2.0-7.44], $\mathrm{I}^{2}=72 \%$ ) ( Fig. $4 \mathrm{a}$ ) or CEA $\geq 192 \mathrm{ng} / \mathrm{mL}$ (OR: $2.94 ; 95 \% \mathrm{Cl}$ : $\left.[1.66-5.21], I^{2}=67 \%\right)(\triangleright$ Fig. $4 b)$. 


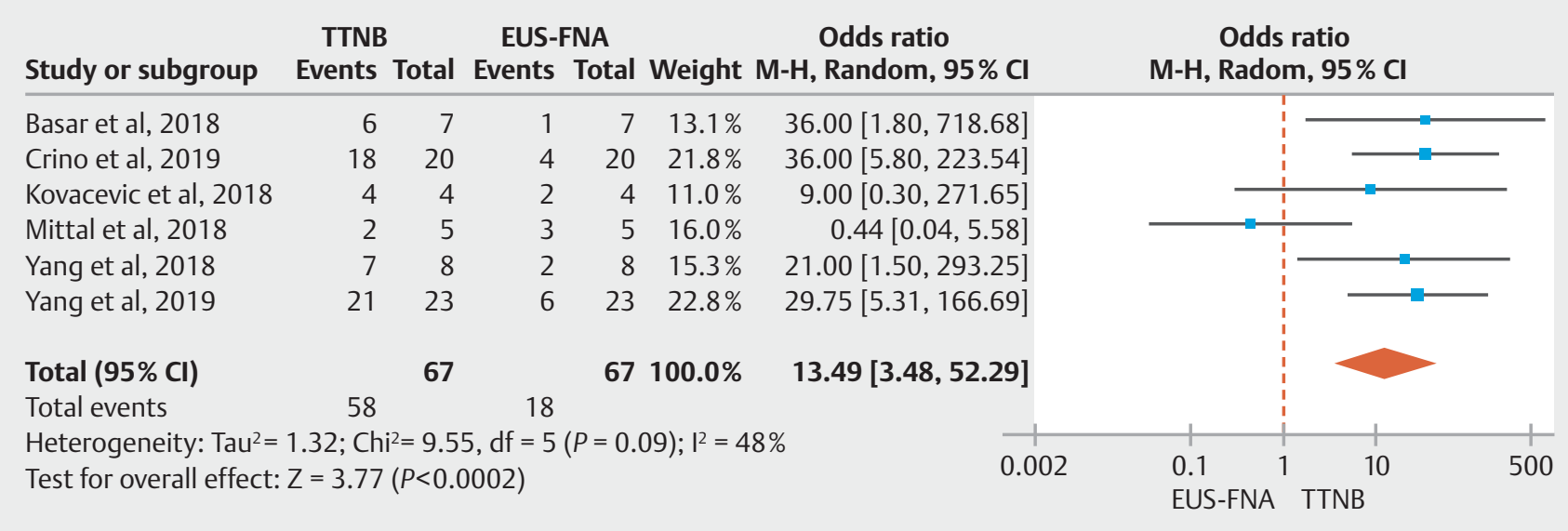

Fig. 5 Pooled concordance of TTNB histology vs FNA cytology with surgical pathology for a specific cyst type (Random-Effect Model).

\section{Serous cystadenoma}

Six studies provided information on serous cystadenoma based on both TTNB histology and FNA cytology [22-27]. Among these studies, the pooled diagnostic yield for a serous cystadenoma was significantly higher with TTNB (12.4\%; $95 \%$ Cl: [7.318.6]) as compared to FNA (1.2\%; $95 \% \mathrm{Cl}$ : [0.3-2.5]). In comparator analysis, the diagnostic yield of a serous cystadenoma was greater with TTNB compared to FNA (OR: 5.35; $95 \% \mathrm{Cl}$ : [2.02-14.16]), with low heterogeneity among the studies $\left(I^{2}=\right.$ 0) ( Fig. 4c).

\section{TTNB and FNA concordance with surgical pathology}

Ninety two patients in the 8 studies included in this meta-analysis underwent surgery for their PCs. Six studies provided information on the respective TTNB histology and FNA cytology for each surgical pathology specimen [22-25, 27, 28].

\section{Diagnosis of specific cyst type}

The pooled concordance of TTNB and FNA with surgical pathology for a specific cyst type were $82.3 \%$ (95\% Cl: [71.9-90.7]) and $26.8 \%$ (95\% Cl: [17.0-37.8]), respectively. Compared to FNA cytology, TTNB histology was significantly more likely (OR: 13.49; 95\% Cl: [3.49-52.29]) to match the diagnosis on surgical pathology; with moderate heterogeneity between the studies $\left(I^{2}=48 \%\right)$ ( Fig. 5$)$.

\section{Diagnosis of mucinous cysts}

The pooled concordance for mucinous cysts was also higher for TTNB (89\%; $95 \%$ Cl: [80.0-95.0]) vs FNA (41\%; $95 \%$ Cl: [2855]). Compared to FNA, TTNB was significantly more likely (OR: 8.93; $95 \% \mathrm{Cl}$ : [1.96-40.77]) to match the diagnosis of mucinous cyst with surgical pathology; with low heterogeneity detected $\left(I^{2}=29 \%\right)$ ( Fig. 6a). Using the surgical pathology as the reference standard, the overall sensitivity and specificity of TTNB for mucinous cysts were $90.1 \%$ (95\% Cl: [78.4-97.6]; $I^{2}=$ $36.5 \%)$ and $94 \%\left(95 \% \mathrm{Cl}:[81.5-99.7] ; \mathrm{I}^{2}=0\right)$, respectively ( Fig.6b, > Fig. 6c).

\section{Diagnosis of histological grading of mucinous cysts}

Four studies provided information on histological grading of mucinous cysts on TTNB histology, FNA cytology, and corresponding surgical pathology $[22,25,27,28]$. The pooled concordance with the histological grade of a mucinous cyst on surgical pathology was significantly higher with TTNB (75.6\%; 95\% Cl: [62.3-86.8]) versus FNA (26\%; 95\% Cl: [6.752.3]). Furthermore, TTNB in comparison to FNA was significantly more likely to match the histologic grade compared to surgical pathology (OR: 10.4; 95\% Cl: [2.93-36.93]); with low heterogeneity $\left(\mathrm{I}^{2}=0\right)(\triangleright$ Fig. 7$)$.

\section{Adverse events}

The pooled rate of AEs was 7.0\% (95\% Cl: [2.3-14.1]; $I^{2}=82.9$ ) ( $\triangleright$ Fig. 8a). The pooled occurrence for intracystic hemorrhage and acute pancreatitis were $5.0 \%\left(95 \% \mathrm{Cl}:[1.2-11.2] ; \mathrm{I}^{2}=\right.$ 82.6) and $2.3 \%\left(95 \% \mathrm{Cl}:[0.5-5.3] ; \mathrm{I}^{2}=62.5\right)$, respectively ( $>$ Fig. 8b, $>$ Fig. $\mathbf{8 c}$ ). None of the cases of intracystic hemorrhage required additional interventions. Among the 10 cases of acute pancreatitis reported, six did not require hospitalization [21, 25,27,28], three were discharged within 24 to 48 hours with supportive care $[25,28]$, whereas one developed a pseudocyst that required endoscopic drainage [27].

\section{Publication bias}

Funnel plots have been included in the supplementary material (Supplementary Fig.9). There was no evidence of substantial publication bias on visual inspection of the funnel plots for any of the analyses except for the calculation of the pooled concordance rate between TTNB, FNA and surgical pathology for a specific cyst type diagnosis, which is in part due to precision bias being skewed by the two larger studies favoring TTNB histology concordance with surgical pathology $[27,28]$.

\section{Discussion}

Accurate diagnosis and risk stratification of PCs is of utmost importance as it may allow the early detection and management of PCs with malignant potential, while limiting unnecessary in- 


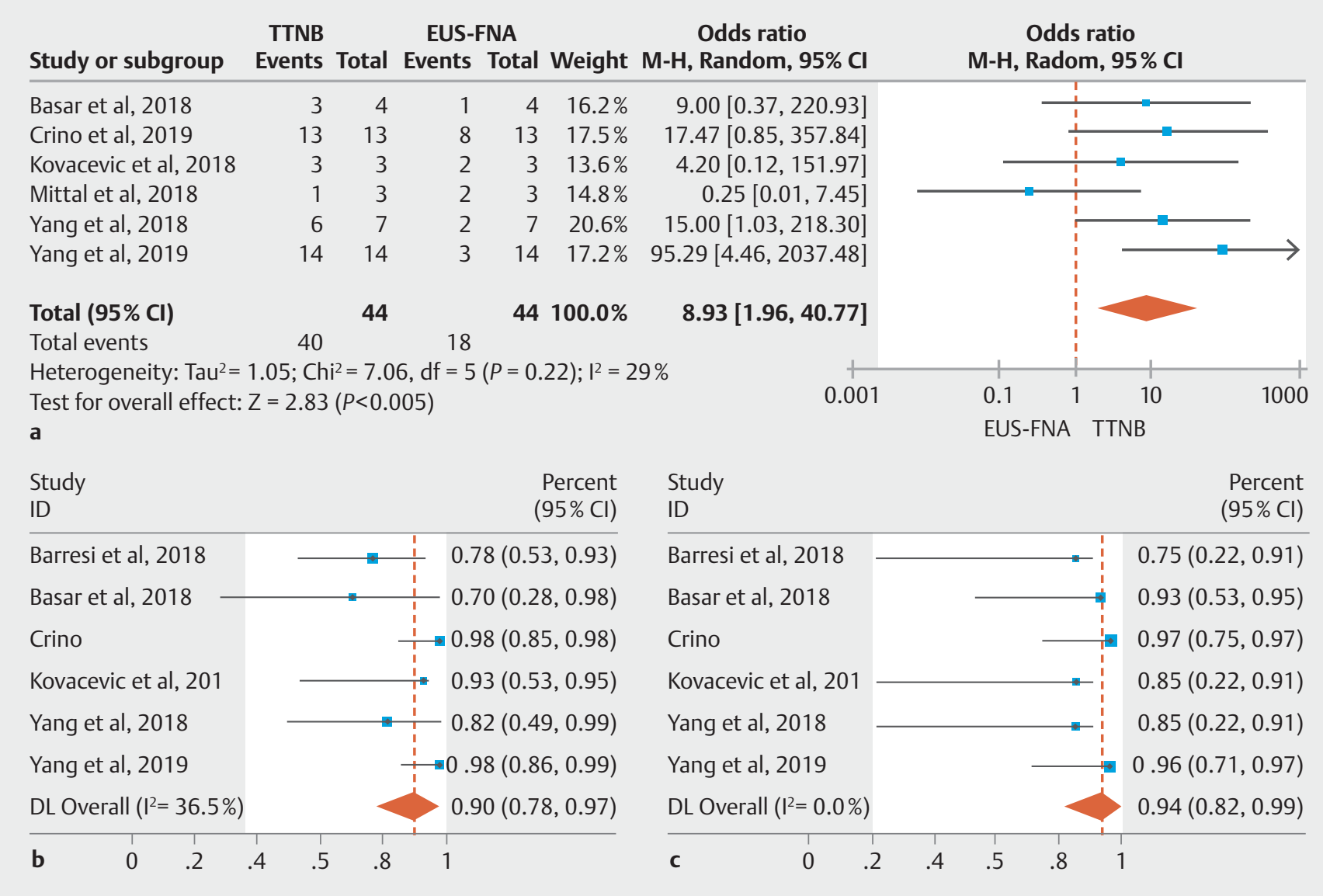

- Fig. 6 a Pooled concordance of TTNB histology vs FNA cytology with surgical pathology for a mucinous cyst (Random-Effect Model). b Pooled sensitivity of TTNB histology for diagnosing a mucinous cyst using surgical pathology as reference standard (Random-Effect Model). c Pooled specificity of TTNB histology for diagnosing a mucinous cyst using surgical pathology as reference standard (Fixed-Effect Model).

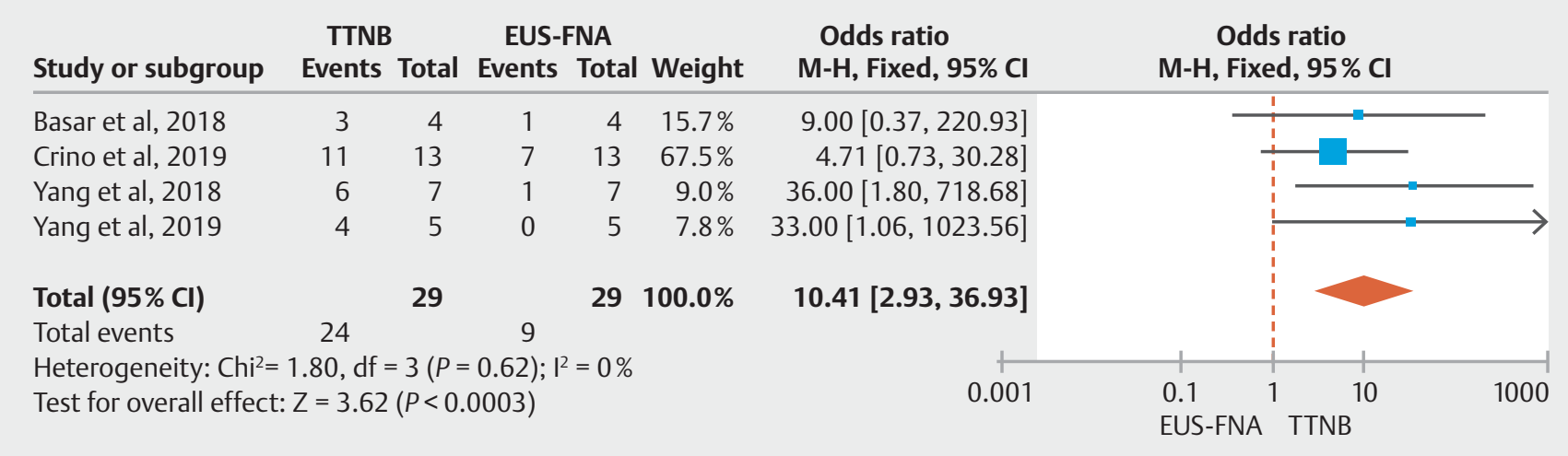

Fig. 7 Pooled concordance of TTNB histology vs FNA cytology with histological grading of mucinous cysts on surgical pathology (FixedEffect Model)

terventions in most inconsequential benign PCs. In this systematic review and meta-analysis, we evaluated the performance of TTNB of PCs using a novel microforceps device.

The overall estimated pooled technical success of TTNB with the microforceps was very high (98.2\%), supporting its applicability in the diagnosis of a broad range of PCs, irrespective of cyst size or location. The microforceps device permits targeted tissue acquisition from the cyst wall, septations and/or mural nodules for histological evaluation, which in turn, potentially increases the likelihood of obtaining a specific cyst diagnosis as compared to FNA cytology. In this meta-analysis, the overall diagnostic yield for a specific cyst type was significantly higher 


\begin{tabular}{|c|c|c|}
\hline $\begin{array}{l}\text { Study } \\
\text { ID }\end{array}$ & & $\begin{array}{r}\text { Percent } \\
(95 \% \mathrm{Cl})\end{array}$ \\
\hline Barresi et al, 2018 & & $0.17(0.08-0.27)$ \\
\hline Basar et al, 2018 & 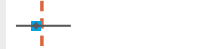 & $0.06(0.01-0.15)$ \\
\hline Crino et al, 2019 & 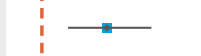 & $0.23(0.14-0.35)$ \\
\hline Kovacevic et al, 2019 & 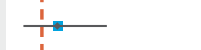 & $0.11(0.03-0.24)$ \\
\hline Mittal et al, 2018 & - & $0.01(0.01-0.08)$ \\
\hline Yang et al, 2018 & - & $0.01(0.01-0.04)$ \\
\hline Yang et al, 2019 & - & $0.01(0.01-0.04)$ \\
\hline Zhang et al, 2018 & & $0.13(0.07-0.19)$ \\
\hline DL Overall $\left(I^{2}=83.0 \%\right)$ & & $0.07(0.02-0.14)$ \\
\hline a & .2 & .8 \\
\hline $\begin{array}{l}\text { Study } \\
\text { ID }\end{array}$ & & $\begin{array}{l}\text { Percent } \\
(95 \% \mathrm{Cl})\end{array}$ \\
\hline Barresi et al, 2018 & & $0.13(0.06-0.23)$ \\
\hline Basar et al, 2018 & & $0.03(0.001-0.11)$ \\
\hline Crino et al, 2019 & 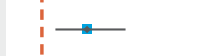 & $0.17(0.09-0.27)$ \\
\hline Kovacevic et al, 2019 & -1 & $0.01(0.01-0.07)$ \\
\hline Mittal et al, 2018 & -1 & $0.01(0.01-0.08)$ \\
\hline Yang et al, 2018 & & $0.03(0.001-0.09)$ \\
\hline Yang et al, 2019 & - & $0.15(0.07-0.27)$ \\
\hline Zhang et al, 2018 & -1 & $0.002(0.002-0.02)$ \\
\hline DL Overall $\left(I^{2}=82.7 \%\right)$ & & $0.05(0.01-0.11)$ \\
\hline b & 0 & .6 \\
\hline $\begin{array}{l}\text { Study } \\
\text { ID }\end{array}$ & & $\begin{array}{r}\text { Percent } \\
(95 \% \mathrm{Cl}) \\
\end{array}$ \\
\hline Barresi et al, 2018 & $\frac{1}{11}$ & $0.004(0.004-0.04)$ \\
\hline Basar et al, 2018 & 1 & $0.006(0.005-0.05)$ \\
\hline Crino et al, 2019 & & $0.04(0.006-0.10)$ \\
\hline Kovacevic et al, 2019 & 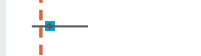 & $0.05(0.002-0.14)$ \\
\hline Mittal et al, 2018 & + & $0.01(0.008-0.08)$ \\
\hline Yang et al, 2018 & & $0.03(0.001-0.1)$ \\
\hline Yang et al, 2019 & $\rightarrow$ & $0.13(0.05-0.23)$ \\
\hline Zhang et al, 2018 & 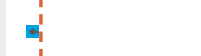 & $0.002(0.002-0.02)$ \\
\hline DL Overall $\left(I^{2}=62.5 \%\right)$ & 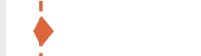 & $0.02(0.009-0.04)$ \\
\hline
\end{tabular}

Fig. 8 a Pooled adverse events with TTNB of pancreatic cysts. b Pooled occurrence of intracystic hemorrhage with TTNB of pancreatic cysts. c Pooled occurrence of acute pancreatitis with TTNB of pancreatic cysts.

with TTNB versus FNA (OR: 9.37; Cl: [5.7-15.4]). The superior diagnostic yield of TTNB for a specific cyst type was further supported in this meta-analysis by the overall higher concordance of TTNB over FNA (OR: 13.49; Cl: [4.5-52.3]) for a specific cyst diagnosis on surgical pathology. Differentiating between specific cyst types has significant clinical implications. For instance, SCAs are benign lesions that do not necessitate surveillance or intervention in the absence of symptoms. However, previous studies have demonstrated that FNA cytology has a low sensitivity for the diagnosis of SCA $[34,35]$. Our results demonstrated that the diagnostic yield for serous cystadenoma was also significantly higher with TTNB as compared to FNA (OR: 5.35; Cl: [2.0-14.2]). In aggregate, these findings suggest that TTNB can be helpful when elucidating a specific cyst type may impact subsequent care.

The limitations of EUS-FNA for the evaluation of mucinous cysts are well-recognized $[4,11,12]$ and additional modalities are much needed to improve our diagnostic ability. In this study, the diagnostic yield for mucinous cysts was significantly higher with TTNB histology when compared to either FNA cytology (OR: 3.86; Cl: [2.0-7.4]) or cyst fluid CEA $\geq 192 \mathrm{ng} / \mathrm{mL}$ (OR: 2.94; Cl:[1.7-5.2]). Using surgical pathology as the reference standard, the pooled sensitivity and specificity of TTNB for mucinous cysts were $90.1 \%$ and $94 \%$, respectively; with low heterogeneity among the studies. Indeed, our data demonstrated that TTNB was nearly 9-fold more likely to diagnose a mucinous cyst when compared to FNA cytology (OR: 8.93; Cl: [2.0-40.8]); and hence, should be entertained as part of the multi-modality approach in the evaluation of PCs.

Accurate risk stratification of mucinous cysts is perhaps the most important yet also the most challenging step in the management of PCs. Cyst fluid analysis is not helpful as neither CEA nor molecular mutations correlate with histological grade. While relatively specific, the diagnostic accuracy of FNA cytology is often hindered by the suboptimal cellular specimen obtained from PCs [11, 36]. In this meta-analysis, we demonstrate that the concordance rate with the histological grade among surgically resected mucinous cysts was 10 -fold higher with TTNB histology when compared to FNA cytology (OR 10.41; Cl: [2.9-37.0]) with low heterogeneity. Given the high rate of tissue adequacy for histological grading, TTNB may prove to add significant value, particularly when triaging patients with no overt "high-risk" cyst features.

The estimated pooled occurrence of AEs associated with TTNB was $7 \%$, with the calculated rate of intracystic hemorrhage and acute pancreatitis being $5 \%$ and $2.3 \%$, respectively. Given the lack of current data to evaluate for risk factors, we can only speculate that the more aggressive mode of tissue acquisition with TTNB may account for the higher rates of adverse events. The study by Crino et al [28] suggests that two TTNB macroscopically visible specimens allowed reaching a $100 \%$ histological adequacy and therefore additional attempts at TTNB may not improve the yield but rather increase the risk of AEs. Further studies are needed to help define not only the optimal number of passes but also the preferred tissue acquisition technique with this device.

This study has several strengths. We performed a systematic literature search that was comprehensive with well-defined inclusion and exclusion criteria, and the quality of the studies was rigorously assessed based on the pre-defined parameters in the modified NOS. All subjects in the included studies underwent both EUS-FNA and TTNB for the comparative outcome meas- 
ures, thereby serving as their own control and reducing variance. Furthermore, it is important to highlight that we strictly used surgical pathology as the reference criterion when calculating the diagnostic accuracy of TTNB for mucinous cysts. We also acknowledge the limitations. Most included studies were small in sample size and retrospective in design, thereby contributing to selection and reporting bias. Furthermore, publication bias was observed for some of the subgroup calculations in this meta-analysis, primarily driven by the small cohort size of each study and the limited number of studies available. In addition, all studies were performed at tertiary care referral centers and thus not representative of the general population. TTNB is not regarded as a standard method in the evaluation of pancreatic cysts, and additional data are needed to further determine its role in the diagnostic algorithm of these lesions. In addition, while our data suggested higher odds of obtaining a correct diagnosis with TTNB as compared to FNA, these results should be interpreted with caution given the large confidence intervals. There was considerable heterogeneity among the studies in the overall analysis comparing diagnostic yield of TTNB vs FNA for specific cyst type, mucinous cyst, and the estimated adverse event rate with TTNB. Possible explanations include variability in: (1) indications for EUS-FNA and TTNB; (2) cyst sampling technique and number of passes for FNA cytology and TTNB histology; (3) pancreas cyst size and morphology; (4) inclusion of cyst fluid analysis and cytology for the evaluation of FNA performance [26]; and (5) patient follow-up postprocedure. We were not able to further evaluate the data based on these parameters given that most studies were inconsistent in the reporting of this information and when available, the outcomes were not categorized according to these findings. Even though a meta-regression analysis could not be performed for these reasons, no evidence of significant heterogeneity was found with regards to the diagnostic accuracy of TTNB for mucinous cysts or in its superiority over FNA in its correlation with a diagnosis of mucinous cyst and histological grade using surgery as reference standard.

\section{Conclusion}

In conclusion, our meta-analysis demonstrates that TTNB has a high sensitivity and specificity for distinguishing mucinous from non-mucinous cysts and may be superior to FNA cytology in risk stratifying mucinous cysts and providing a specific cyst diagnosis. Future well-designed comparative studies between TTNB and FNA in the evaluation of PCs are needed to corroborate these results.

\section{Competing interests}

Dr. Draganov is a consultant for Boston Scientific, Olympus, Cook Medical, Lumendi and Microtech. Dr. Yang is a consultant for US Endoscopy, Boston Scientific, Lumendi and Steris.

\section{References}

[1] Lee KS, Sekhar A, Rofsky NM et al. Prevalence of incidental pancreatic cysts in the adult population on MR imaging. Am J Gastroenterol 2010; 105: 2079-2084

[2] Khashab MA, Kim K, Lennon AM et al. Should we do EUS/FNA on patients with pancreatic cysts? The incremental diagnostic yield of EUS over CT/RMI for prediction of cystic neoplasms Pancreas 2013; 42: 717-721

[3] Visser BC, Yeh BM, Qayyum A et al. Characterization of cystic pancreatic masses: relative accuracy of CT and MRI. AJR Am J Roentgenol 2008; 189: 648-656

[4] Alkaade S, Chahla E, Levy M. Role of endoscopic ultrasound-guided fine-needle aspiration, cytology, viscosity, and carcinoembryonic antigen in pancreatic cyst fluid. Endosc Ultrasound 2015; 4: 299-303

[5] Brugge WR. The use of EUS to diagnose cystic neoplasms of the pancreas. Gastrointest Endosc 2009; 69: S203-\$209

[6] Brugge WR, Lewandrowski K, Lee-Lewandrowski E et al. Diagnosis of pancreatic cystic neoplasms: a report of the cooperative pancreatic cyst study. Gastroenterology 2004; 126: 1330-1336

[7] Vege SS, Ziring B, Jain R et al. American Gastroenterological Association Institute Guideline on the Diagnosis and Management of Asymptomatic Neoplastic Pancreatic Cysts. Gastroenterology 2015; 148: 819-822

[8] Tanaka M, Fernandez-del-Castillo C, Kamisawa T et al. Revisions of international consensus Fukuoka guidelines for the management of IPMN of the pancreas. Pancreatology 2017; 17: 738-753

[9] Elta GH, Enestvedt BK, Sauer BG et al. ACG Clinical Guideline: Diagnosis and Management of Pancreas Cysts. Am J Gastroenterol 2018; 113: 464-479

[10] European Study Group on Cystic Tumours of the Pancreas. European evidence-based guidelines on pancreatic cystic neoplasms. Gut 2018; 67: 789-804

[11] de Jong K, Poley JW, van Hooft JE et al. Endoscopic ultrasound-guided fine-needle aspiration of pancreatic cystic lesions provides inadequate material for cytology and laboratory analysis: initial results from a prospective study. Endoscopy 2011; 43: 585-590

[12] Attasaranya S, Pais S, LeBlanc J et al. Endoscopic ultrasound-guided fine needle aspiration and cyst fluid analysis for pancreatic cysts. JOP 2007; 8: 553-563

[13] Brugge WR, Lewandrowski K, Lee-Lewandrowski E et al. Diagnosis of pancreatic cystic neoplasms: a report of the cooperative pancreatic cyst study. Gastroenterology 2004; 126: 1330-1336

[14] Park WG, Mascarenhas R, Palaez-Luna M et al. Diagnostic performance of cyst fluid carcinoembryonic antigen and amylase in histologically confirmed pancreatic cysts. Pancreas 2011; 40: 42-45

[15] Gaddam S, Ge PS, Keach JW et al. Suboptimal accuracy of carcinoembryonic antigen in differentiation of mucinous and nonmucinous pancreatic cysts: results of a large multicenter study. Gastrointest Endosc 2015; 82: 1060-1069

[16] Samarasena JB, Nakai Y, Shinoura S et al. EUS-guided, through-theneedle forceps biopsy: a novel tissue acquisition technique. Gastrointest Endosc 2015; 81: 225-226

[17] Coman RM, Schlachterman A, Esnakula AK et al. EUS-guided throughthe-needle forceps: clenching down the diagnosis. Gastrointest Endosc 2016; 84: 372-373

[18] Attili F, Pagliari D, Rimbas M et al. Endoscopic ultrasound-guided histological diagnosis of a mucinous non-neoplastic pancreatic cyst using a specially designed through-the-needle microforceps. Endoscopy 2016; 48: E188-E189

[19] Huelsen AX, Cooper CX, Saad NX et al. Endoscopic ultrasound-guided, through-the-needle forceps biopsy in the assessment of an inci- 
dental large pancreatic cystic lesion with prior inconclusive fine-needle aspiration. Endoscopy 2017; 49: E109-E110

[20] Kovacevic B, Karstensen JG, Havre RF et al. Initial experience with EUSguided microbiopsy forceps in diagnosing pancreatic cystic lesions: A multicenter feasibility study (with video). Endosc Ultrasound 2018; 7: 383-388

[21] Barresi L, Crino SF, Fabbri C et al. Endoscopic ultrasound-through-theneedle biopsy in pancreatic cystic lesions: A multicenter study. Dig Endosc 2018; 30: 760-770

[22] Basar O, Yuksel O, Yang D et al. Feasibility and safety of micro-forceps biopsy in the diagnosis of pancreatic cysts. Gastrointest Endosc 2018; 88: 79-86

[23] Kovacevic B, Klausen P, Hasselby JP. A novel endoscopic ultrasoundguided through-the-needle microbiopsy procedure improves diagnosis of pancreatic cystic lesions. Endoscopy 2018; 50: 1105-1111

[24] Mittal C, Obuch JC, Hammad H et al. Technical feasibility, diagnostic yield, and safety of microforceps biopsies during EUS evaluation of pancreatic cystic lesions (with video). Gastrointest Endosc 2018; 87: 1263-1269

[25] Yang D, Samarasena JB, Jamil LH et al. Endoscopic ultrasound-guided through-the-needle microforceps biopsy in the evaluation of pancreatic cystic lesions: a multicenter study. Endosc Int Open 2018; 6: E1423-E1430

[26] Zhang ML, Arpin RN, Brugge WR et al. Moray micro forceps biopsy improves the diagnosis of specific pancreatic cysts. Cancer Cytopathol 2018; 126: 414-420

[27] Yang D, Trindade AJ, Yachimski P et al. Histologic analysis of endoscopic ultrasound- guided through the needle microforceps biopsies accurately identifies mucinous pancreas cysts. Clin Gastroenterol Hepatol 2019; 17: 1587-1596
[28] Crino SF, Bernardoni L, Brozzi L et al. Association between macroscopically visible tissue samples and diagnostic accuracy of EUS-guided through-the-needle microforceps biopsy of pancreatic cystic lesions. Gastrointest Endosc 2019; 90: 933-943

[29] Stang A. Critical evaluation of the Newcastle-Ottawa scale for the assessment of the quality of nonrandomized studies in meta-analyses. Eur J Epidemiol 2010; 25: 603-605

[30] Guyatt GH, Oxman AD, Kunz R et al. GRADE guidelines: 7. Rating the quality of evidence - inconsistency. J Clin Epidemiol 2011; 64: 12941302

[31] Easterbrook PJ, Gopalan R, Berlin JA et al. Publication bias in clinical research. Lancet 1991; 337: 867-872

[32] Lau J, loannidis JP, Terrin $N$ et al. The case of the misleading funnel plot. BMJ 2006; 333: 597-600

[33] Higgins JPT, Green S. Cochrane Handbook for Systematic Review of Interventions. Chichester, West Sussex; Hoboken NJ: John Wiley \& Sons; 2011

[34] Lilo MT, VandenBussche C], Allison BD et al. Serous cystadenoma of the pancreas: potentials and pitfalls of a preoperative cytopathologic diagnosis. Acta Cytol 2017; 61: 27-33

[35] Belsey NA, Pitman MB, Lauwers GY et al. Serous cystadenoma of the pancreas: limitations and pitfalls of endoscopic ultrasound-guided fine-needle aspiration biopsy. Cancer 2008; 114: 102-110

[36] Pitman MB, Centeno BA, Genevay M et al. Grading epithelial atypia in endoscopic ultrasound fine-needle aspiration of intraductal papillary mucinous neoplasms: an international observer concordance study. Cancer Cytopathol 2013; 121: 729-736 